\title{
The Toulmin Model Today: Introduction to the Special Issue on Contemporary Work using Stephen Edelston Toulmin's Layout of Arguments
}

\section{DAVID HITCHCOCK}

\author{
Department of Philosophy \\ McMaster University \\ Hamilton, Ontario \\ Canada \\ L8S $4 K 1$ \\ E-mail:hitchckd@mcmaster.ca \\ and
}

\section{BART VERHEIJ}

\author{
Kunstmatige Intelligentie \\ Rijksuniversiteit Groningen \\ Grote Kruisstraat 2/1 \\ 9712 TS Groningen \\ The Netherlands \\ E-mail:b.verheij@ai.rug.nl
}

In 1958, Stephen Edelston Toulmin's The Uses of Argument saw the light of day. This book has never been out of print; an updated second edition appeared in 2003. It has influenced, and continues to influence, researchers in speech communication and philosophy, and also in artificial intelligence, cognitive psychology and application domains such as law and medicine.

In The Uses of Argument Toulmin emphasized a number of points that are by now familiar, but still deserve attention:

1. Reasoning and argument involve not only support for points of view, but also attack against them.

2. Reasoning can have qualified conclusions.

3. There are other good types of argument than those of standard formal logic.

4. Unstated assumptions linking premisses to a conclusion are better thought of as inference licenses than as implicit premisses.

5. Standards of reasoning can be field-dependent, and can themselves be the subject of argumentation.

Each of these points is illustrated by his famous, graphically schematized layout of arguments. The element of rebuttal illustrates the first point, the qualifier shows the second point, and his views on warrant and backing demonstrate the last three points. 
Modern work on topics such as defeasible reasoning, argumentation schemes and field-dependent standards of reasoning has roots in Toulmin's ideas. Toulmin has also strongly influenced the graphical representation of argument today, e.g. in software. It is the goal of this special issue to show the breadth of the influence of Toulmin's The Uses of Argument by bringing together current research from a variety of perspectives. The issue contains eight research papers. Four of the papers (those by Aberdein, Freeman, Hitchcock and Reed \& Rowe) were presented in May 2005 at a conference entitled 'The uses of argument' at McMaster University, sponsored by the Ontario Society for the Study of Argumentation. A selection of papers from this conference about Toulmin's work on argumentation, along with some other papers on the same topic, is to be published as a volume in the Argumentation Library; this volume will include the papers in the present issue.

In 'A Citation-Based Reflection on Toulmin and Argument', Ronald P. Loui uses citation counts to measure the influence of Toulmin's work. He reports that citations in the leading journals in the social sciences, humanities and science and technology put Toulmin and his works in the top 10 among philosophers of science and philosophical logicians of the 20th century. Thus, he concludes, Toulmin's Uses of Argument, and Stephen Toulmin's work in general, have been essential contributions to twentieth century thought.

In 'Translating Toulmin Diagrams: Theory Neutrality in Argument Representation', Chris Reed and Glenn Rowe address the task of translating between two diagrammatic systems for diagramming arguments: Toulmin's six-component layout and the box-arrow diagram introduced by Beardsley (1950) and refined by Thomas (1977). They address with 'engineering pragmatism' such old and deep theoretical challenges as the relationship between Toulminian warrants and their counterparts in box-and-arrow diagrams. They adopt from the artificial intelligence literature solutions to such other challenges as how to allow Toulmin diagrams to be recursive. And they develop solutions with 'both practical utility and theoretical interest' to new challenges such as the relationship between refutations, rebuttals and undercutters, and the role of multiple warrants.

Three papers in this issue consider the applicability of Toulmin's model to particular fields of argumentation.

Toulmin's layout was originally developed on a jurisprudential model, in conscious opposition to the mathematical model then standard in logic (Toulmin, 1958, p. 95). It turns out however that it can be applied even in mathematics. In 'The Uses of Argument in Mathematics', Andrew Aberdein shows how Toulmin's model can be used to lay out the reasoning in mathematical proofs. He argues that incom- 
patible reconstructions characteristically reflect real disagreement about the nature of the proof.

In 'AI \& Law, Logic and Argument Schemes', Henry Prakken argues that recent artificial intelligence research modeling various components of legal reasoning has used the approach of argumentation schemes, with their associated critical questions. While this research has gone beyond the Toulmin model, it has taken to heart at least three lessons from Toulmin: the different roles of argument premisses, the defeasibility of everyday reasoning, and the field-dependence of standards for evaluating argument.

In 'Toulmin's Model and the Solving of Ill-Structured Problems', James F. Voss reports on the use of the Toulmin model to analyze verbal protocols of the solving of ill-structured problems. The analysis of lines of reasoning as long as 10 paragraphs uncovered few examples of warrants and encountered difficulties in distinguishing datum from backing. The Toulmin model did not capture the components of the problem-solving process, for which a higher level of analysis was required. The Toulmin analysis of micro-arguments was fitted into the components of this higher level. At a still more general level, however, the overall problem-solving process was considered to be a Toulmintype structure.

Another three papers deal with questions of evaluation within the context of the Toulmin model.

In 'Systematizing Toulmin's Warrants: An Epistemic Approach', James B. Freeman addresses the problem of evaluating warrants. Toulmin himself proposed to classify warrants by field and to look for the backing for a given warrant in the field to which the warrant belonged. Freeman finds this approach problematic, both because it is often difficult to assign a warrant to a field and because leaving standards of evaluation up to the practitioners of each field threatens an unacceptable relativism. Instead, Freeman proposes to classify warrants, construed as claims of general connections, according to how we discover and ultimately justify them. There turn out to be four main types of warrants: a priori, empirical, institutional and evaluative. Freeman illustrates the differences by contrasting empirical physical warrants with institutional warrants.

In 'Evaluating Arguments Based on Toulmin's Scheme', Bart Verheij proposes a system for evaluating arguments laid out according to a formal reconstruction of Toulmin's model. This formalization, and the resulting system of evaluation, are inspired by work in the field of artificial intelligence on the formalization of argumentation. Among other innovations, Verheij adds two more types of rebuttals to the three types recognized by Toulmin. The resulting evaluation system provides for three evaluation statuses for the components of Toulmin's 
scheme: justified, unevaluated, defeated. Further, the system is dialectical and non-monotonic, in the sense that an attack on a previously justified component of an argument can defeat it. But this component can be reinstated, through an attack on the rebuttal.

Toulmin proposed his model as a way of making candid the components of an argument construed as a defense of an assertion (1958, p. 12). In 'Good Reasoning on the Toulmin Model', David Hitchcock maintains that the model can also be applied to solo reasoning. $\mathrm{He}$ proposes guidelines for solo verbal reasoning in which a reasoner aims to arrive at a correct answer to a question from information at the reasoner's disposal: justified and adequate grounds, justified warrant, justified assumption that no defeaters apply. He proposes criteria for fulfillment of each of these four conditions.

The eight papers were selected from seventeen submissions using peer review, and were revised in the light of that review. We would like to thank the following referees for helping us with the selection process: Trevor Bench-Capon, Peter Cramer, Chris Eisenhart, James Freeman, Michael Gilbert, Tom Gordon, Leo Groarke, Dale Hample, Anthony Hunter, Ralph Johnson, Ronald Leenes, Ronald P. Loui, Peter McBurney, Daniel O'Keefe, Fabio Paglieri, Henry Prakken, Phyllis Rooney, Guillermo Simari, Christina Slade, Olaf Tans, Tim van Gelder, James Voss, Douglas Walton, John Woods.

\section{REFERENCES}

Beardsley, M. C.: 1950, Practical Logic, Prentice-Hall, Englewood Cliffs, NJ.

Thomas, S. N.: 1977, Practical Reasoning in Natural Language, Prentice-Hall, Englewood Cliffs, NJ.

Toulmin, S. E.: 1958, The Uses of Argument, Cambridge University Press, Cambridge. 